\title{
Analysis on Removal of Vehicle Shadow Based on Edge Feature and HSV Color Space
}

\author{
Wang Yangping ${ }^{1,2^{*}}$, Zhu Zhengping ${ }^{3}$, Sui Jing ${ }^{1}$, Wang Song ${ }^{1}$ and Sun Yue ${ }^{4}$ \\ ${ }^{1}$ School of Electronic \& Information Engineering, Lanzhou Jiaotong University, Lanzhou 730070, China \\ ${ }^{2}$ Experiment Center on Computer Science, Lanzhou Jiaotong University, Lanzhou 730070, China \\ ${ }^{3}$ School of Information Engineering, Lanzhou City College, Lanzhou 730070, China \\ ${ }^{4}$ Department of International Development, Community, and Environment, Clark University, Worcester, MA 01610, United States
}

Received 11 May 2017; Accepted 16 October 2017

\begin{abstract}
In complex lighting environments, vehicle shadows in video surveillance images severely affect the detection and tracking of vehicles and the recognition of abnormal vehicle behavior. Edge-based shadow removal algorithms remove most parts of the shadow, but they leave fine shadow edges unremoved. Meanwhile, hue-saturation-value (HSV) color space-based shadow removal algorithms may erroneously recognize vehicles as shadows and remove them if a vehicle color is similar to that of the shadow. To address this problem, a shadow removal method that fuses edge-based and HSV color space-based algorithms was proposed. Most of the shadows were first removed by an edge-based shadow removal algorithm, in which subtractions and differential operations were performed between the current frame and background image based on a background model. Then, an exclusive OR (XOR) operation was conducted. The image from the HSV approach was then fused with the image produced by the edge-based method. Finally, mathematical morphology was used to process the combined images, thereby improving the efficacy of shadow removal. Experimental results prove that the proposed shadow removal algorithm can effectively remedy the defects of simple shadow removal algorithms. In normal illumination conditions, the detection rate and resolution of this method can exceed $90 \%$. This study provides an excellent method for optimizing the removal of moving object shadows through the use of multiple feature fusion in video surveillance images, especially in complex lighting environments.
\end{abstract}

Keywords: Video image, Moving object detection, Edge feature, HSV color space, Shadow removal

\section{Introduction}

The increase of motor vehicles highlights the traffic jam on the road, and the annual amount of traffic accidents hovers at a high level. Especially on the highway, drivers' parking and lane changing in defiance of the Highway Code easily lead to congestion, thereby lowering driving efficiency and severely influencing normal travel of people. In an effort to detect vehicles that change lanes, prevent congestion, relieve traffic pressure, and decrease unnecessary traffic accidents in an effective and timely manner, the detection of abnormal traffic behavior based on video images has become a main research topic for intelligent transportation systems. Vehicle detection and recognition through video surveillance serve as the main premise of vehicle classification and tracking, which enhance vehicle detection systems. However, in complex environments (e.g., outdoor illumination), shadows are detected as moving objects depending on the size and shape of the tracked vehicle. As a result, some regions of the vehicle are obtained inaccurately, thereby resulting in loss of information. In other cases, the multiple regions of a vehicle are fused as a single image, thereby hindering vehicle tracking [1,2]. Halkarnikar et al. [3] explained that the appearance of shadow usually interferes with the extraction and measurement of a vehicular target's geometric parameters, which then leads to misjudgments in target

*E-mail address: w_yp73@163.com

ISSN: 1791-2377 C 2017 Eastern Macedonia and Thrace Institute of Technology. All rights reserved. doi:10.25103/jestr.105.10 detection, non-implementation of vehicle tracking, and abnormal behavior detection.

At present, the detection and removal of vehicle shadows in traffic scenes have become a key technology for intelligent vehicle target detection and tracking systems. Several works have proposed detection and removal algorithms based on shadow models and feature characterization [4-9]. Theoretical studies and practical applications of these algorithms play an important role in the development of intelligent transportation systems. Nonetheless, the above methods are still limited by certain inadequacies. In particular, model-based methods are only suitable for certain types of scenes and cannot be applied universally. These algorithms are also complex and time consuming, making it difficult to apply in real-time scenarios. Vehicle shadow detection based on singular characteristics (e.g., color, edge, geometric shape, and texture) suffer from high false-positive rates when used in complex lighting conditions.

In this study, shadow edge features are fused with color space features. The multiple yet complementary sources of information can be used for the removal of shadows, thereby improving detection rate and resolution of algorithms for abnormal vehicle behavior detection.

\section{State of the art}

Shadow removal algorithms for vehicle shadows have been proposed. Methods based on edge detection were applied to 
Wang Yangping, Zhu Zhengping, Sui Jing, Wang Song and Sun Yue/

\section{Journal of Engineering Science and Technology Review 10 (5) (2017) 84-89}

vehicle shadow removal [10,11], but they could not completely remove the fine shadow edges. Amato et al. [12] used the invariance of shadow textures to eliminate vehicle shadows. However, false detection rates for vehicles with unclear textural characteristics are relatively high. Choi et al. [13] designed a three-layer cascade shadow estimator using the maximum likelihood method to estimate color and luminance thresholds. However, the algorithm was susceptible to color interference and the candidate shadow regions were highly pixelated, which affected the estimations. Jiang et al. [14] proposed an adaptive method based on the edge pixel sampling statistics. Despite commendable detection accuracies, insufficient sampling was observed when edge pixels were low. Salvador et al. [15] presented a color-invariant shadow feature method that segmented shadow regions effectively. However, the algorithm was complex and cost inefficient, and the process was affected by changes in environmental illumination during real-time detection. In the study conducted by Cucchiara et al. [16], shadow areas were detected based on hue information of the hue-saturation-value(HSV) color space. Excellent recognition was obtained, but this approach could not distinguish shadow areas from dark foregrounds.

Tian et al.[17] used RGB color information to detect static shadows. Detection rates exceeded $90 \%$, but the shadow areas of high-speed motion could not be recognized. In the study performed by Cavallaro et al.[18], adaptive background difference thresholds were obtained by modeling the Gaussian noise of an image, after which shadow detection was conducted using the same thresholds. The approach could adapt to changes in illumination, but the image sequences processed using this method must conform strictly to Gaussian noise distributions. Li et al.[19] proposed a shadow detection method that fused textural features and shadow attributes. The varying patterns of pixel textures before and after they were covered by shadows were established, after which the values of the local binary pattern operator for the pixels in the current and background frames were differentiated to detect the candidate shadow regions. However, this method was not very effective for regions with blurry textural features. Zhang et al. [20] proposed a shadow removal method that combined the $\mathrm{YCbCr}$ color space with the Gaussian mixture model (GMM). The differences between the foreground and background of the moving regions in $\mathrm{YCbCr}$ were analyzed to acquire the motion characteristics of the shadows, thereby facilitating the development of a Gaussian mixture shadow model. Both distribution characteristics of the shadows and the Gaussian probabilities were fused for shadow removal. However, to establish the shadow model, the method relied on the GMM algorithm, which required several calculations, thereby limiting real-time detection. Yu et al. [21] utilized HSV color space information for foreground and background frames to remove shadows. However, when vehicle color was similar to the background color, the algorithm treats the vehicle color as a shadow and removes it from the image, in which results in incomplete moving objects.

In summary, using single feature-based methods for vehicle shadow detection and removal is impractical because of the complexities in traffic and illumination condition, similarities between vehicle and shadow colors, and indistinct vehicle textural features in traffic video images. Hence, to address the aforementioned weaknesses in shadow removal algorithms, this study proposes a shadow removal method that fuses an edge-based algorithm with an HSV color space-based algorithm. In this method, the shadow of the moving object detected by a background subtraction method is taken as the object for research and is preliminary calculated by the edge-based algorithm. The detected image from the edge-based algorithm is then fused with that from the HSV algorithm. Finally, mathematical morphology is employed to process the fused results and achieve improved shadow removal efficacies. The proposed method resolves incomplete removal of shadows by the edge-based algorithm and the incomplete detection of shadows by the color spacebased algorithm.

The remainder of this study is organized as follows: Section 3 describes the proposed method for vehicle shadow removal in traffic video images, in which edge features are fused with HSV color space. Section 4 discusses the applicability of the method through case studies. Section 5 presents the conclusions.

\section{Methodology}

\subsection{Detection of moving objects}

Extracting the moving object (foreground) serves as the basis for analyzing the motion state of vehicles; it is a prerequisite for vehicle tracking and behavior analysis. This study adopts the averaging method to establish a background model. The background subtraction method [16,17] is used to extract the foreground, as shown in Equations (1) to (4).

$$
\begin{aligned}
& B_{i}(x, y)=\frac{1}{N} \sum_{i=1}^{N} f_{i}(x, y) \\
& B_{i+1}=(1-\alpha) B_{i}(x, y)+\alpha f_{i}(x, y) \\
& D_{i}(x, y)=\left|f_{i}(x, y)-B_{i-1}(x, y)\right| \\
& F(x, y)= \begin{cases}1 & D_{i}(x, y) \geq t h \\
0 & D_{i}(x, y)<t h\end{cases}
\end{aligned}
$$

where $f_{i}(x, y)$ is the original image; $B_{i}(x, y)$ refers to the background image; $D_{i}(x, y)$ represents the difference image; and $F(x, y)$ is the binary image of the foreground. $\alpha=0.005, N=200$, and $t h=45$ are used to detect a vehicle in a video sequence of an extensively used highway (i.e., Highway1, https://cvrr.ucsd.edu/aton/shadow/). The detection result for the 18th frame is shown in Fig. 1.

\subsection{Shadow detection and removal}

Multiple vehicle shadows in the moving object region may be detected erroneously by the background subtraction method when size and shape vehicle features vary. Such as is the case for moving objects in Fig. 1 (c), in which the presence of a shadow causes the three vehicles to look like a single vehicle. This error can be explained by the same motion characteristics of the shadow and the vehicle, which make it appear that the shadow is a moving object, thereby leading to inaccurate vehicle tracking. This study combines the edge feature with HSV color space to remove shadows and extract moving objects. 


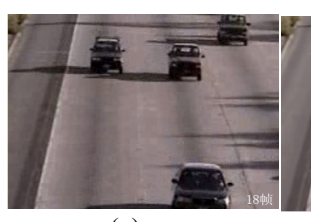

(a)

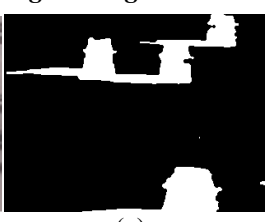

(c)

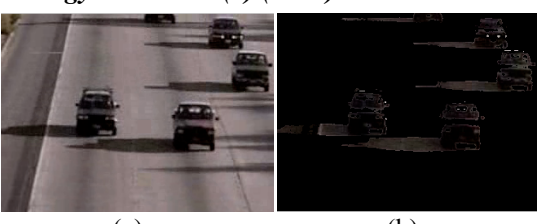

(a)

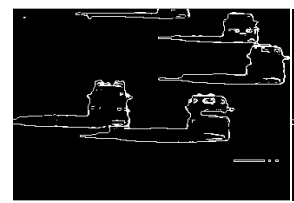

(d)

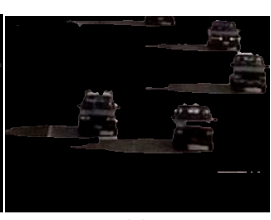

(c)

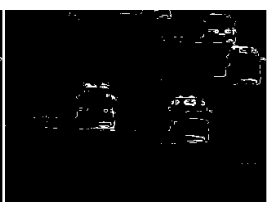

(f)

The edges of an image contain rich information and deserve much attention in image analysis (i.e., structure and attributes). The outline of a vehicle can be obtained by detecting the edges of a moving object. Shadow edges are clearer and more concise than vehicle edges, and the marginal density of shadows is smaller than that of vehicles. Methods based on existing edge detection algorithms detect the edges of the moving objects obtained by background subtraction. In the present study, the detection results are subjected to exclusive OR (XOR) operation to derive the final effect of shadow removal.

The study adopts the Laplacian-of-Gaussian (LoG) edge detection operator $[18,19]$ to detect the edges of moving objects. The algorithm flow is shown in Fig. 4 (a). Initially, the LoG method smoothens the original image to suppress noise to the largest extent. The edges of the smoothened image are then extracted. The operation can be described in three steps: (1) the grayscale image is convolved using the two-dimensional Gaussian function to smoothen the grayscale image; (2) the smoothened image is reinforced by the two-dimensional Laplacian operator; and (3) edges are detected according to zero crossing of the second-order derivative. The above third step focuses on extracting the trajectory of the zero crossing for the reinforced secondorder directional derivative image, as shown in Equations (7) and (8).

$$
\begin{aligned}
& \nabla^{2}=\partial^{2} / \partial x^{2}+\partial^{2} / \partial y^{2} \\
& G(x, y)=\frac{1}{2 \pi \sigma} \exp \left(-\frac{x^{2}+y^{2}}{2 \sigma^{2}}\right) \\
& \operatorname{img}(x, y)=G(x, y) * f(x, y) \\
& g(x, y)=\nabla^{2}\{i m g(x, y)\}=\nabla^{2}[G(x, y) * f(x, y)]=\nabla^{2} G(x, y) * f(x, y) \\
& \nabla^{2} G(x, y)=\left[\frac{x^{2}+y^{2}-2 \sigma^{2}}{\sigma^{4}}\right] \exp ^{\left(-\frac{x^{2}+y^{2}}{2 \sigma^{2}}\right)}
\end{aligned}
$$

where the Laplacian operator $\nabla^{2}$ is used to process the smoothened image, as shown in Equation (5). Equation (6) is a two-dimensional Gaussian function, in which $\sigma$ is the standard deviation of the Gaussian function and $*$ is the convolution operator. Equation (9) defines the LoG operator. A $3 \times 3$ Gaussian filter with $\sigma=0.25$ is combined with the edge-based shadow removal algorithm to remove the shadow of moving objects detected from the Highwayl video. The shadow removal result for the 18th frame is shown in Fig. 2.

(a) The 18th frame image; (b) Foreground subtracted by background; (c) Foreground divided by background; (d) Foreground edge of (b); (e) Foreground edge of (c); (f) Shadow removal result.

Fig. 2. Experimental result of edge-based shadow removal.

\subsubsection{Shadow removal based on HSV color space}

In the video frame sequence obtained by a camera, the brightness for any pixel $i$ at a certain image frame can be expressed by Equation (10).

$$
V(i)=E(i) * p(i)
$$

where $E(i)$ is illumination intensity. In addition, $p(i)$ is the reflection coefficient of the object surface related to object properties,which is fixed for the same material.The illumination intensity $E(i)$ can be obtained by Equation (11).

$$
E(i)= \begin{cases}C_{A}+C_{p} \cos \angle(N(i), L) & \text { Light } \\ C_{A} & \text { Shadow }\end{cases}
$$

where $C_{A}$ and $C_{p}$ represent the intensity of the illuminant and the diffuse reflection, respectively; $N(i)$ is the normal direction of object surface; and $L$ is the vector from the object surface in the illuminant direction. The luminance ratio for pixel $i$ before and after shadow appearance can be expressed by Equation (12).

$$
Q(i)=\frac{C_{A}}{C_{A}+C_{p} \cos \angle(N(i), L)}
$$

When an image belongs to the same frame, the intensity of the illuminant and diffuse reflection is equal. From Equation (12), the brightness ratio before and after shadow appearance is smaller than 1 , i.e., $Q(i)<1$.

HSV color model reserves precise gray information and hue information to highlight the shadow difference between the moving object and the background. HSV emulates the color discrimination of a human eye. Hence, the method adopts HSV color spaces to detect and recognize shadows and non-shadows. According to the above theory of illumination, the shadow region does not exhibit significant changes in hue $(\mathrm{H})$ and saturation $(\mathrm{S})$ relative to the foreground region in HSV color spaces, but its brightness (V) is rather different because the shadow region is much darker than the non-shadow region. We removed shadow for this reason with the algorithms in Equations (13) to (16). The algorithm flow is shown in Fig. 4 (b). 
Wang Yangping, Zhu Zhengping, Sui Jing, Wang Song and Sun Yue/

Journal of Engineering Science and Technology Review 10 (5) (2017) 84-89

$$
\Delta V=\frac{V_{f}(x, y)}{V_{b}(x, y)}
$$

$$
\Delta S=\left|S_{f}(x, y)-S_{b}(x, y)\right|
$$

$\Delta H=\left|H_{f}(x, y)-H_{b}(x, y)\right|$

$F(x, y)= \begin{cases}255 & \alpha \leq \Delta V \leq \beta \text { I } \Delta S \leq T_{s} \text { I } \Delta H \leq T_{h} \\ 0 & \text { others }\end{cases}$

Where $H_{f}, S_{f}$, and $V_{f}$ refer to the foreground hue, saturation, and brightness, respectively, while $H_{b}, S_{b}$, and $V_{b}$ are the background hue, saturation, and brightness, respectively. Then, $\alpha=0.05, \beta=0.2, T_{s}=30$, and $T_{h}=15$. The HSV color space-based shadow removal algorithm is used to remove the shadow of the moving objects detected from an actual highway video sequence (i.e., Highway-R). The shadow removal effect of the 36th frame is shown in Fig. 3.

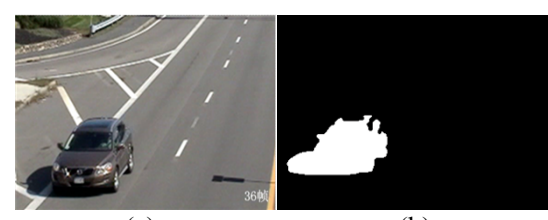

(a) (b)

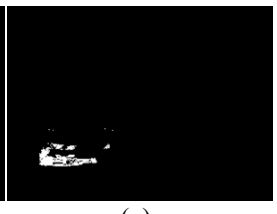

(c)
Fig.3. Result of shadow removal based on HSV color spaces. (a) Original image of the 36th frame; (b) Binarized foreground; (c) Shadow removal result.

3.2.2 Shadow removal that fuse edge-based and color space-based algorithms

LoG detection operator was employed to detect the edges of moving objects obtained using background subtraction in the study. XOR operation was also performed on the detected results, and the final effect of shadow removal was obtained (Fig. 2). In the figure, the edges of common shadows were eliminated by XOR operation (i.e., value $=0$, inverse value $=1$ ) for the image segmented by two thresholds. Then, the outline of the vehicle with fine shadow edges was obtained. However, the shadow was not removed completely. For moving objects obtained by background subtraction in HSV color spaces, the shadow region was similar for the nonshadow region in terms of hue and saturation, but their brightness differed considerably. Therefore, different thresholds were set for hue, saturation, and brightness to constrain the detected shadow region, remove the shadow, and derive the shadow removal result (Fig. 3). The shadow removal algorithm removed the shadow fully but wrongly detected the vehicle as a shadow and subsequently removed it when vehicle color was the same as the shadow color, thereby leading to an incomplete detection. As shown in Fig. 3 (c), only a part of the information of vehicle was detected.

Based on the introduction, analysis, and comparison of the two shadow removal algorithms, the edge-based approach removed most of the shadows although some of the fine edges were remained. In contrast, the HSV color space-based shadow removal algorithm eliminated the shadow region fully, but it mistakenly removed shadows when the colors of the shadow and the vehicle were the same. The method combined the relative merits of the two algorithms for shadow removal. Vehicle 1 represents the edge-based algorithm. Vehicle 2, which was obtained using the HSV color space-based shadow removal algorithm, was applied with an OR operation for morphological processing to complete the vehicle detection. The flowchart is shown in Fig. 4 (c).

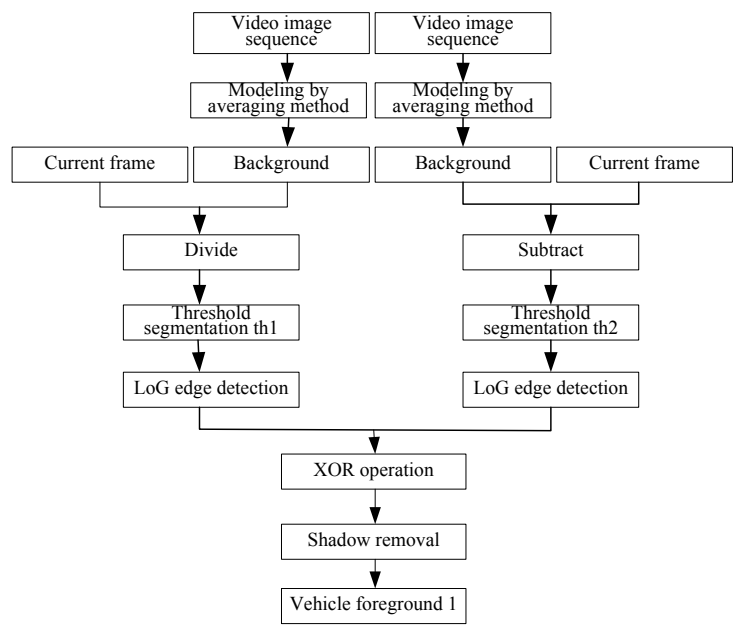

(a)

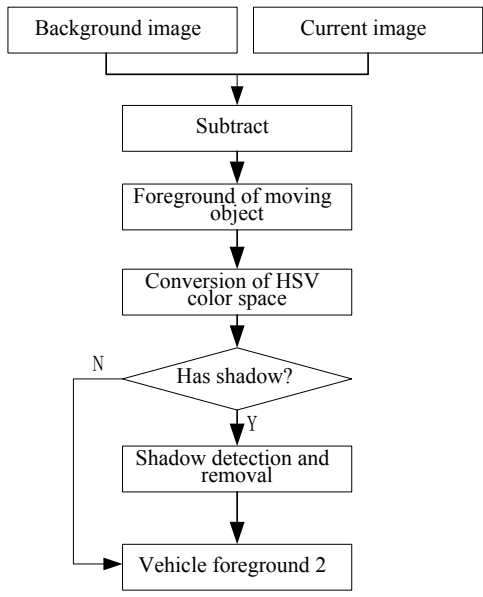

(b)

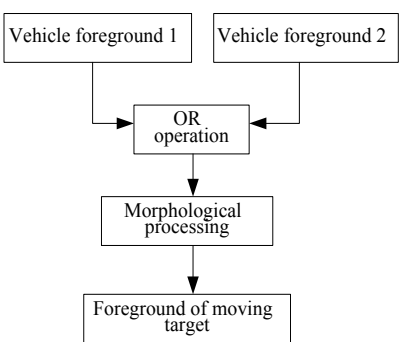

(c)

(a) Edge-based shadow removal; (b) HSV color space-based shadow removal; (c) Fused edge-based and HSV color space-based shadow removal. Fig. 4. Flowcharts of shadow removal algorithms.

\section{Results Analysis and Discussion}

The detection algorithm for this experiment was conducted using Matlab2014b on a computer with Pentium (R) DualCore CPU at 2.60 GHZ and memory of 4GB. Experiments were conducted on an extensively used benchmark shadow detection motion image sequence (Highway1 and Highway2 from https://cvrr.ucsd.edu/aton/shadow/) and an actual highway image sequence (Highway-R). Subsequently, the 
Wang Yangping, Zhu Zhengping, Sui Jing, Wang Song and Sun Yue/

Journal of Engineering Science and Technology Review 10 (5) (2017) 84-89

performance of the improved shadow removal algorithm was verified. The sequences of the test image are listed in Tab.1. Results comparisons on the experimental effects are shown in Fig. 5.

Table 1. Sequences of test image

\begin{tabular}{l|l|l|l}
\hline & Highway1 & Highway2 & $\begin{array}{c}\text { Highway- } \\
\text { R }\end{array}$ \\
\hline $\begin{array}{l}\text { Length of image } \\
\text { sequence(frame) }\end{array}$ & 440 & 500 & 547 \\
$\begin{array}{l}\text { Size of image(pixel) } \\
\text { Size of shadow }\end{array}$ & $320 * 240$ & $320 * 240$ & $320 * 240$ \\
Shadow intensity & Large & Small & Medium \\
\hline
\end{tabular}
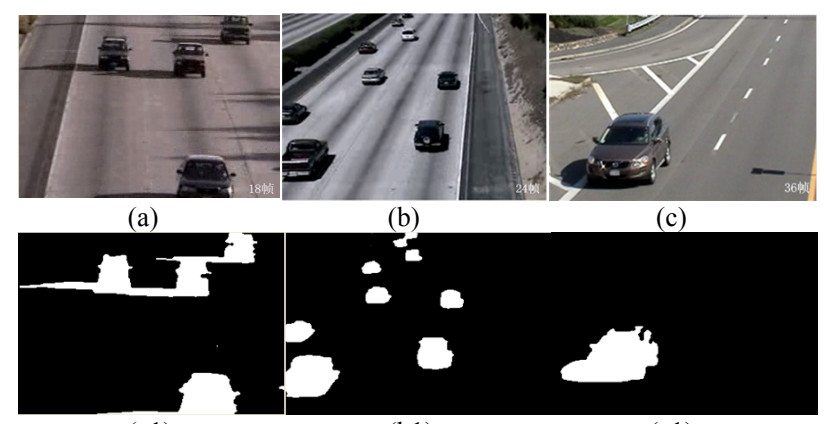

(a1)
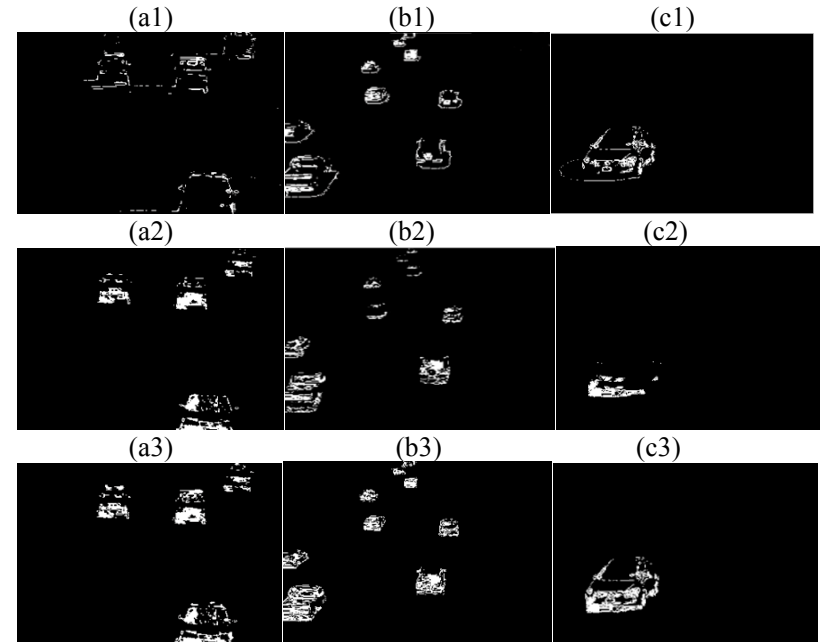

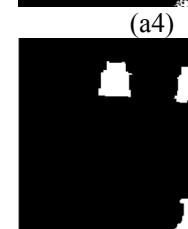

(a5)

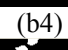

(b4)
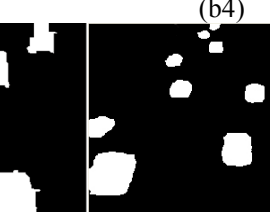

(a) Original image of Highway1; (b) Original image of Highway2; (c) Original image of Highway-R; (a1) Foreground binarization for Highway1; (b1) Foreground binarization for Highway2; (c1) Foreground binarization for Highway-R; (a2) Edge-based shadow removal for Highway1; (b2) Edge-based shadow removal for Highway2; (c2) Edge-based shadow removal for Highway-R; (a3) HSV color space-based shadow removal for Highway1; (b3) HSV color spacebased shadow removal for Highway2; (c3) HSV color space-based shadow removal for Highway-R; (a4) Shadow removal for Highway1; (b4) Shadow removal for Highway2; (c4) Shadow removal for Highway-R; (a5) Morphological processing for Highway1; (b5) Morphological processing for Highway2;(c5) Morphological processing for Highway-R.

Fig.5. Comparison results of experimental effects among shadow removal algorithms.

Experimental results for the binarized foreground of video sequences in Figs. 4 (a1), 5 (b1), and 5 (c1) showed that each frame appeared with a vehicle shadow. Several vehicles were easily recognized as a single vehicle when the shadow was large, as shown by the binarized image of the moving objects with a shadow in Fig.4 (a1). The edge-based algorithm removed most of the shadow, but some fine edges remained, as shown in Figs. 5 (a2), 5 (b2), and 5 (c2). When vehicle color differed from the background color, the algorithm could detect and completely remove the shadow well (i.e., vehicle detection is not affected), as shown in Figs. 5 (a3) and 5 (b3). In contrast, when vehicle color was the same as the road color, the HSV algorithm recognized the similarly colored vehicles as a vehicle with a shadow. What appeared as shadows (i.e., in actual, another vehicle) was removed, thereby leading to incomplete vehicle detection. As shown in Fig. 5 (c3), given that the vehicle color was the same as the road color, only part of vehicle was reserved while most other parts were mistaken as a shadow and were thus removed. The combination of the two methods could detect the moving object completely and remove the shadow fully, as shown in Figs. 5 (a4), 5 (b4), and 5 (c4). The above detection result indicates that the shadow removal algorithm eliminates the drawbacks of simple shadow removal algorithms by combining and supplementing two algorithms, thereby removing the shadow fully without affecting vehicle detection and recognition.

We adopted performance evaluation formulas [20], as shown in Equations (17) and (18), to determine the results of different algorithms and further show the superiority of the method. The larger the shadow detection rate $\eta$ and shadow resolution $\xi$ are, the better the effect of shadow removal. The final detection result is listed in Tab. 2.

$$
\begin{gathered}
\eta=\frac{T P_{S}}{T P_{S}+F N_{S}} \\
\xi=\frac{T P_{F}^{\prime}}{T P_{F}+F N_{F}}
\end{gathered}
$$

where $T P_{S}$ is the quantity of recognized shadow pixels; $F N_{S}$ refers to the quantity of unrecognized shadow pixels; $T P_{F}$ is the quantity of recognized vehicle pixels; $F N_{F}$ pertains to the quantity of unrecognized vehicle pixels; and $T P_{F}{ }^{\prime}$ is the pixel difference between the vehicle pixel and vehicle pixel wrongly detected as a shadow.

Table 2. Detection results of shadow removal algorithms (\%)

\begin{tabular}{l|c|c|c|c|c|c}
\hline \multirow{2}{*}{\multicolumn{1}{c|}{ Algorithm }} & \multicolumn{2}{|c|}{ Highway1 } & \multicolumn{2}{c|}{ Highway2 } & \multicolumn{2}{c}{ Highway-R } \\
\cline { 2 - 7 } & $\boldsymbol{\eta}$ & $\boldsymbol{\xi}$ & $\boldsymbol{\eta}$ & $\boldsymbol{\xi}$ & $\boldsymbol{\eta}$ & $\boldsymbol{\xi}$ \\
\hline $\begin{array}{l}\text { Edge-based shadow } \\
\text { removal algorithm }\end{array}$ & 54.67 & 95.18 & 47.31 & 98.76 & 66.24 & 97.04 \\
$\begin{array}{l}\text { HSV-based shadow } \\
\text { removal algorithm }\end{array}$ & 97.21 & 60.11 & 98.31 & 40.21 & 96.22 & 51.65 \\
$\begin{array}{l}\text { Improved Shadow } \\
\text { removal algorithm }\end{array}$ & 86.21 & 90.15 & 77.21 & 96.78 & 92.35 & 92.10 \\
\hline
\end{tabular}

Experimental results are presented in Tab.2. The shadow detection rate $\eta$ of the proposed algorithm was lower than that of the HSV color space-based shadow removal algorithm, but greater than that of the edge-based shadow removal algorithm. The shadow resolution $\xi$ was also lower than that of the edge-based algorithm, but greater than that of the HSV algorithm. Considering the shadow resolution and shadow detection rate, the suggested algorithm significantly improves the shadow detection rate at the expense of a small amount of shadow resolution relative to the edge-based shadow removal algorithm. While relative to the HSV-based algorithm, the algorithm achieves a large increase in shadow resolution at the expense of a small amount of shadow detection. 


\section{Conclusions}

A novel method that fused the edge-based algorithm with the HSV color space-based algorithm was developed to remove vehicle shadows in the images and detect moving vehicle targets and then identify the abnormal behavior of vehicles in video surveillance images under complex light environment. The following conclusions could be drawn:

(1) Shadow removal algorithms based on single-edge features have low shadow detection rates but high shadow resolution. Shadow removal algorithms based on single HSV color features have high shadow detection rates but low shadow resolution. Therefore, complementation of these features in the proposed algorithm could improve the detection rates and resolution of surveillance images.

(2) In terms of overall shadow resolution and shadow detection rates, the proposed algorithm significantly improved shadow detection rates at the expense of a small amount of shadow resolution for the edge-based shadow removal algorithm. In comparison with the HSV color space-based algorithm, the proposed algorithm achieved more significant increase in shadow resolution with small cost in shadow detection rate.

Based on the experimental results, the detection rate and resolution of the proposed algorithm varied considerably in different illumination environments. In normal lighting conditions, the detection rate and resolution of this algorithm could exceed $90 \%$.

The algorithm proposed in this work combined edges and color features for the detection and removal of shadows, thereby resolving the incomplete removal of fine shadow edges for edge-based shadow removal algorithms, and the misrepresentation caused by similar vehicle-shadow colors for color space-based shadow removal algorithms. Overall, the proposed algorithm significantly improved the shadow removal efficacies. The findings of this research can serve as reference for the removal of moving object shadows in video surveillance images and the accurate identification of abnormal vehicle behavior. However, different lighting environments will significantly affect the detection rate and resolution of this algorithm, which implies that its robustness requires further improvement. In future studies, additional feature-related information, such as geometry and texture, will be incorporated into the shadow removal method to detect moving vehicles more accurately than the current setup, as well as to provide technical support for subsequent image processing steps.

\section{Acknowledgements}

The study was supported by the Program for Changjiang Scholars and Innovative Research Team in Universities (Grant No. IRT_16R36) and the Science and Technology Project of Gansu Colleges and Universities (Grant No. 2017D-08).

Access article distributed under the terms of the Creative Commons Attribution Licence

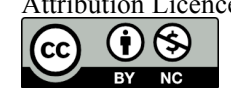

\section{References}

1. Nijad Al-Najdawi, Helmut E. Bez, Jyoti Singhai, Eran.A. Edirisinghe, "A survey of cast shadow detection algorithms". Pattern Recognition Letters, 33(6),2012,pp.752-764.

2.Andres Sanin, Conrad Sanderson, Brian C. Lovell, "Shadow detection:a survey and comparative evaluation of recent methods". Pattern Recognition, 45(4),2012,pp.1684-1695.

3. PP Halkarnikar,SN Talbar, PN Vasambekar, "Object separation in shadows clutter in video sequences". International Conference on Radar, Communication and Computing, 2012, pp.299-302.

4. SH Khan, M Bennamoun, F Sohel, R Togneri., "Automatic shadow detection and removal from a single image". IEEE Transactions on Pattern Analysis \& Machine Intelligence, 38(3),2016,pp.431-446.

5. A Movia, A Beinat, F Crosilla, "Shadow detection and removal in RGB VHR images for land use unsupervised classification". ISPRS Journal of Photogrammetry and Remote Sensing, 119,2016,pp.485495.

6. Xiang Jinhai,Fan Heng, Liao Honghong, "Moving object detection and shadow removing under changing illumination condition". Mathematical Problems in Engineering,2014,2014,pp.1-10.

7. Zhu Zhou,Lu Xiaobo,Wei Pengm,Zeng Weili, "Vehicle shadow detection algorithm based on superpixel and SVM".Journal of Southeast University(Natural Science Edition) ,45(3),2015,pp.443447.

8. Qi Meibin, Pan Yan, Zhang Yinxia, "Preceding moving vehicle detection based on shadow of chassis". Journal of Electronic Measurement and Instrument,26(1),2012,pp.54-59.

9. Kaushik Kaushik, Deb,Animesh Kar,et al, "Cast shadow detection and removal of moving objects from video based on HSV color space”. SmartCR, 5(1),2015,pp.38-50 .

10. Li Haoliang, Shui Qinghe, Fan Wenbing, Yang Xiaonan, "A novel vehicles shadow removal method based on edge detection".Journal of Zhengzhou University(Engineering Science). 35(5),2014,pp.1114.

11. Jiang $\mathrm{Ke}, \mathrm{Li}$ Aihua, Su Yanzhao, "An adaptive shadow detection algorithm using edge texture and sampling deduction".Journal of Xi'an Jiaotong University, 47(2),2013,pp.39-46.

12. Amato A, Mozerov M G, Bagdanov A D, et al. "Accurate moving cast shadow suppression based on local color constancy detection". IEEE Transactions on Image Processing, 20(10),2011, pp.29542966.

13. JinMin Choi, Yung Jun Yoo, Jin Young Choi,"Adaptive shadow estimator for removing shadow of moving object".Computer Vision and Image Understanding,114(9),2010,pp. 1017 -1029.
14. Jiang Ke ,Li Aihua, Cui Zhigao, “Adaptive shadow detection using global texture and sampling deduction". IET Computer Vision, 7(2), 2013, pp.115-122.

15. Salvador E.Cavallaro A. Ebrahimi T, "Cast Shadow Segmentation Using Invariant Color Features". Computer Vision and Image Understanding, 95(2),2004,pp.238-259.

16. Cucchiara R, Piccardi M,Prati A, "Detecting Moving Objects and Shadow in Video Streams". IEEE Transactions on Pattern Analysis and Machine Intelligence, 2(10),2003,pp.1337-13442.

17. Tian Jiandong ,SunJing, Tang Yandong, "Tricolor Attenuation Model for Shadow Detection". IEEE Transactions on Image Processing, 18(10),2009,pp.2355-2363.

18. Cavallaro A,Salvador E, Ebrahimi T, "Shadow-aware object-based video processing”.IEEE Proceedings on Vision,Image and Signal Processing, 152(4),2005,pp.398-406.

19. Li Han, Wu Qisheng, "Shadow detection method with color correlation and texture difference".Computer Engineering, 37(15), 2011,pp.146-148.

20. Zhang Hongying, Li Hong, Sun Yigang, "Shadow removal algorithm based on Gaussian mixture model".Journal of Computer Applications,33(1),2013,pp.31-34.

21. Yu Wanxia, Du Taihang, "Video based vehicle detection based on background difference in color space". Computer Simulation, 1,2010 , pp.285-287.

22. Tibor Trnovszký, Peter Sýkora, "Róbert Hudec.Comparison of background subtraction methods on near infra-red spectrum video sequences". Procedia Engineering, 192,2017,pp. 887-892.

23. Yiqin Cao, Zhangming Lei, Xiaosheng Huang, Zhen Zhang, Tao Zhong, "A vehicle detection algorithm based on compressive sensing and background subtraction". Aasri Procedia, 1,2012,pp.480-485.

24. He Qiang, Yan Li, "Algorithm of edge detection based on LOG and canny operator". Computer Engineering, 37(3),2011,pp.210-212.

25. Şaban Öztürk, Bayram Akdemir,Prati, A,Mikic, I,Trivedi, M.M,et al, "Comparison of edge detection algorithms for texture analysis on glass production". Procedia-Social and Behavioral Sciences, 195,2015, pp.2675-2682.

26. Andrea Prati,Ivana Mikic, Mohan M. Trivedi, "Detecting moving shadows: algorithms and evaluation". IEEE Transactions on Pattern Analysis and Machine Intelligence, 25(7),2003,pp. 918923. 